\title{
Cooperation vs. Hierarchy: An Information-theoretic Comparison
}

\author{
Lalitha Sankaranarayanan \\ WINLAB, Department of ECE \\ Rutgers University \\ Piscataway, NJ 08854 \\ Email: lalitha@winlab.rutgers.edu
}

\author{
Gerhard Kramer \\ Bell Laboratories \\ Lucent Technologies \\ Murray Hill, NJ 07974 \\ Email: gkr@bell-labs.com
}

\author{
Narayan B. Mandayam \\ WINLAB, Department of ECE \\ Rutgers University \\ Piscataway, NJ 08854 \\ Email: narayan@winlab.rutgers.edu
}

\begin{abstract}
The performance of source-cooperation in a multiaccess network is compared to that of using a wireless relay. The former network is modeled as a multi-access channel with generalized feedback and the latter as a multi-access relay channel. Using power as the cost metric, achievable rates and outage probabilities for the two networks are compared under a total transmit power constraint and a specified geometry. The use of a relay is shown to be advantageous for a variety of wireless fading channels.
\end{abstract}

\section{INTRODUCTION}

Cooperation in communication networks results when terminals use their power, time, and bandwidth resources to mutually enhance their transmissions. Cooperation can be induced in several ways, and we consider two approaches. First, we allow source nodes to forward data for each other and second, we introduce wireless relay nodes when cooperation between source nodes is either undesirable or not possible. We refer to networks employing the former approach as cooperative networks and those employing the latter as hierarchical networks.

There are important differences between cooperative and hierarchical networks that are not easy to analyze from an information-theoretic point of view. For example, in cooperative networks one likely needs economic incentives to induce cooperation [1]. On the other hand, hierarchical networks incur infrastructure costs [2]. While incentives and infrastructure costs are important elements that need to be considered in comparing the two networks, we use only the total transmit power as the cost metric for our comparisons.

The paper is organized as follows. In Section II, we present the network models. The cooperative network is modeled as a multi-access channel with generalized feedback (MAC-GF) [3], [4] and the hierarchical network as a multi-access relay channel (MARC) [5]-[7]. We place half-duplex constraints on the transmit-receive capabilities of all nodes. In Section III, we develop partial decode-and-forward (PDF) strategies for both networks. Finally, in Section IV, we compare the rate and diversity gains for the two networks under the PDF strategy and a total average transmit power constraint.

\section{Channel And Network Models}

\section{A. Network Model}

Our networks consist of $M$ source nodes numbered $1,2, \ldots, M$ and a destination node $M+1$. For the MARC there is one additional node (the relay node $M+2$ ). The input and output alphabets of node $m$ are the respective $\mathcal{X}_{m}$ and $\mathcal{Y}_{m}$. We write $\mathcal{S}=\{1,2, \ldots, M\}$ and $x_{\mathcal{G}}=\left\{x_{m}: m \in \mathcal{G}\right\}$ for $\mathcal{G} \subseteq \mathcal{S}$. For the MARC, we write $\mathcal{T}=\{1,2, \ldots, M-1, M, M+2\}$ for the set of transmitters.

Let $X_{m, i} \in \mathcal{X}_{m}$ be the input of node $m$ at time $i$. For complex Gaussian channels, the output of node $m$ at time $i$ is

$$
Y_{m, i}=\left(\sum_{k \neq m} h_{m, k, i} X_{k, i}\right)+Z_{m, i}
$$

where the $Z_{m, i}$ are independent, proper, complex, zero-mean, unit variance Gaussian noise random variables, and $h_{m, k, i}$ is the fading gain between transmitter $k$ and receiver $m$ at time $i$. Note that $X_{M+1, i}=0$ for both the MAC-GF and the MARC. The transmitted signals have average block power constraints

$$
\sum_{i=1}^{n} E\left|X_{m, i}\right|^{2} \leq n P_{m} \quad m \in \mathcal{T} .
$$

The above model allows the network nodes to transmit and receive simultaneously, which may not possible in practice. We therefore impose a half-duplex constraint on every node, i.e., each node can be in one of two states, listen or transmit (LoT) [8]. We then have, for all $m$ and $i$,

$$
Y_{m, i}=0 \quad \text { if } X_{m, i} \neq 0 .
$$

We define $\sigma_{m, i} \in\{L, T\}$ to be the $L o T$ state at node $m$ and time $i$. We assume that the $\sigma_{m, i}$ are known universally to all nodes at all times.

\section{B. Cooperative Network}

We model the cooperative network as a Gaussian MAC-GF. The average block power at the $m^{t h}$ source node is $P_{m}^{(C)}$ and is subject to the constraint (2), i.e., $P_{m}^{(C)} \leq P_{m}$. We refer to the half-duplex MAC-GF as the HD-MAC-GF.

There are many ways to duplex $M$ sources over two states. We here divide the total time into $M+1$ slots. In slot $t$, 
$t=1,2, \ldots, M$, only source $t$ transmits. In slot $M+1$, all sources transmit. Let $\alpha_{t}$ denote the fraction of time allocated to slot $t$. Note that the HD-MAC-GF can be viewed as a MACGF with $(M+1) L o T$ states where the probability of the $t^{t h}$ state is $\alpha_{t}$.

\section{Hierarchical Network}

We model the hierarchical network as a Gaussian MARC with $M+1$ inputs $X_{m, i}, m \in \mathcal{T}$, and two outputs $Y_{M+1, i}$ and $Y_{M+2, i}$ given by (1) [7]. We write $P_{m}^{(H)}$ for the average block power in (2), so that $P_{m}^{(H)} \leq P_{m}$.

A half-duplex MARC, or HD-MARC, results when the relay listens for a fraction $\alpha$ of the total time (or bandwidth) and transmits for the remaining fraction. We refer to the fraction $\alpha$ as the listen $(L)$ slot and the fraction $(1-\alpha)$ as the talk (T) slot. Note that the HD-MARC can be viewed as a MARC with two LoT states.

\section{Network Power Constraint}

We assume that the HD-MARC sources only transmit. Similarly, the destination node $M+1$ in both the HD-MAC-GF and the HD-MARC only listens. We will additionally impose a network-wide power constraint of

$$
\sum_{k=1}^{M} P_{k}^{(C)}=\sum_{k \in \mathcal{T}} P_{k}^{(H)} \leq P_{t o t} .
$$

\section{E. Fading Model}

We model the fading gains as

$$
h_{m, k, i}=\frac{A_{m, k, i}}{\sqrt{d_{m, k}^{\gamma}}}
$$

where $d_{m, k}$ is the distance between the $m^{\text {th }}$ receiver and the $k^{t h}$ source, $\gamma$ is the path-loss exponent, and $A_{m, k, i}$ is a proper complex fading random variable. We consider three kinds of fading channels in this paper.

1) no fading $A_{m, k, i}=1$ for all $m, k, i$.

2) ergodic phase-fading with $A_{m, k, i}=e^{j \theta_{m, k, i}}$ where $\theta_{m, k, i}$ are jointly independent and identically distributed (i.i.d) uniformly between $[-\pi, \pi)$ for all $i$.

3) Rayleigh quasi-static (slow) fading where $A_{m, k, i}$ are jointly i.i.d zero-mean, unit variance proper, complex Gaussian random variables.

\section{Cooperative Strategies}

Three commonly used cooperative communication strategies are decode-and-forward (DF), compress-and-forward (CF), and amplify-and-forward (AF) [5], [7], [9], [10]. The $\mathrm{DF}, \mathrm{CF}$, and $\mathrm{AF}$ strategies result when the cooperating node or relay decodes, compresses or amplifies, respectively, its received signal before forwarding to the destination. The DF strategy can be generalized to become a partial decode-andforward (PDF) strategy where the relay decodes only one of two message streams from each source [11, theorem 7]. We here consider the PDF strategy and numerically compare the two networks with $M=2$ source nodes. However, for completeness we next describe strategies for $M \geq 2$ source nodes.

\section{A. Cooperative Networks}

Willems presents a coding strategy for the MAC-GF with $M=2$ in [3, chap. 7]. For a network of $M>2$ sources, there is a combinatorial explosion in the number of code constructions due to the potential to broadcast messages to any subset of the sources. For simplicity, we assume that all nodes cooperate to transmit the messages they decode from all other nodes. We denote the messages at node $m$ by $w_{m 1}$ and $w_{m 2}$, where $w_{m 1}$ and $w_{m 2}$ take on $2^{n R_{m 1}}$ and $2^{n R_{m 2}}$ values, respectively. We use codewords $\underline{x}_{m}\left(w_{m 1}\right.$, $\left.w_{m 2}, w_{0}\right), \underline{v}_{m}\left(w_{m 2}, w_{0}\right)$, and $\underline{u}\left(w_{0}\right)$ to transmit $w_{m 1}, w_{m 2}$, and a cooperative message $w_{0}$, where $w_{m 0}$ takes on $2^{n R_{m 0}}$ values. The stream $w_{m 2}$ is decoded by all sources while the destination decodes all streams. Let $\mathcal{G}^{c}$ be the complement of $\mathcal{G}$ in $\mathcal{S}, R_{\mathcal{G}, j}=\sum_{m \in \mathcal{G}} R_{m, j}, j=1,2$, and $R_{\mathcal{G}}=R_{\mathcal{G}, 1}+R_{\mathcal{G}, 2}$. We use backward decoding at the destination [3] to prove the following theorem.

Theorem 1: The rate tuple $\left(R_{1}, R_{2}, \ldots, R_{M}\right)$ is achievable for an $M$-source MAC-GF if, for all $\mathcal{G} \subseteq \mathcal{S}$, we have

$$
\begin{aligned}
R_{\mathcal{G}, 2} & \leq \min _{m \in \mathcal{G}^{c}}\left\{I\left(V_{\mathcal{G}} ; Y_{m} \mid X_{m}, U, V_{\mathcal{G}^{c}}\right)\right\} \\
R_{\mathcal{G}, 1} & \leq I\left(X_{\mathcal{G}} ; Y_{M+1} \mid X_{\mathcal{G}^{c}}, V_{\mathcal{S}}, U\right) \\
R_{\mathcal{S}} & \leq I\left(X_{\mathcal{S}} ; Y_{M+1}\right)
\end{aligned}
$$

where the joint probability distribution function of the random variables factors as

$$
p(u) \cdot\left[\prod_{m=1}^{M} p\left(x_{i}, v_{i} \mid u\right)\right] \cdot p\left(y_{\mathcal{S}}, y_{M+1} \mid x_{\mathcal{S}}\right) .
$$

The code construction for the MAC-GF can be applied to the HD-MAC-GF model as the partial decode-and-forward (PDF) strategy. Recall that we are now restricting attention to strategies where only node $m$ transmits during slot $m$ for $m=1,2, \ldots, M$. We obtain the following rate bounds.

Theorem 2: The rate tuple $\left(R_{1}, R_{2}, \ldots, R_{M}\right)$ is achievable for an $M$-source HD-MAC-GF if, for all $\mathcal{G} \subseteq \mathcal{S}$, we have

$$
\begin{aligned}
R_{m, 2} & \leq \min _{k \in[1, M], k \neq m}\left\{\alpha_{m} I\left(V_{m} ; Y_{k}\right)\right\} \\
R_{\mathcal{G}, 1} & \leq\left(\begin{array}{c}
\sum_{m \in \mathcal{G}} \alpha_{m} I\left(X_{m} ; Y_{M+1} \mid V_{m}\right)+ \\
\alpha_{M+1} I\left(X_{\mathcal{G}} ; Y_{M+1} \mid X_{\mathcal{G}^{c}}, U\right)
\end{array}\right) \\
R_{\mathcal{S}} & \leq \sum_{m=1}^{M} \alpha_{m} I\left(X_{m} ; Y_{M+1}\right)+\alpha_{M+1} I\left(X_{\mathcal{S}} ; Y_{M+1}\right)
\end{aligned}
$$

where the joint probability distribution of the random variables factors as

$$
p\left(u, \sigma_{\mathcal{S}}\right) \cdot\left[\prod_{m=1}^{M} p\left(x_{i}, v_{i} \mid u, \sigma_{\mathcal{S}}\right)\right] \cdot p\left(y_{\mathcal{S}}, y_{M+1} \mid x_{\mathcal{S}}, \sigma_{\mathcal{S}}\right) .
$$

Remark 3: The above bounds can also be obtained from (6) $-(8)$ by conditioning on the $(M+1)$ LoT states. 
For a Gaussian HD-MAC-GF, consider proper complex Gaussian inputs. The resource allocation problem then involves optimizing over the

- time fractions $\alpha_{m}$

- power tuples $\left(P_{1}^{(C)}, P_{2}^{(C)}, \ldots, P_{M}^{(C)}\right)$,

- fractions $\gamma_{m, t}$ of power $P_{m}^{(C)}$ transmitted by the source $m$ in time slot $t$, and

- fractions $\beta_{m, t}$ of power $\gamma_{m, t} P_{m}^{(C)}$ allocated by the source $m$ to transmitting $w_{m 1}$.

\section{B. Hierarchical Networks}

Consider the PDF strategy where each source sends two messages in any $n$-length block. Thus, the source $m$ transmits $w_{m 1}$ and $w_{m 2}$ in each block using codewords $\underline{x}_{m}\left(w_{m 1}\right.$, $\left.w_{m 2}, w_{m 0}\right), \underline{v}_{m}\left(w_{m 2}, w_{m 0}\right)$, and $\underline{q}_{m}\left(w_{m 0}\right)$, where $w_{m 0}$ is the message transmitted cooperatively with the relay via the relay's codeword $\underline{x}_{M+2}\left(w_{10}, w_{20} \ldots, w_{M 0}\right)$. As before, $w_{m j}$ takes on $2^{n R_{m j}}$ values. In each block, the relay decodes the current $w_{m 2}, m=1,2, \ldots, M$, while the destination decodes $\left(w_{m 1}, w_{m 0}\right)$. The $m^{t h}$ source rate is $R_{m}=R_{m, 1}+R_{m, 2}$. We use backward decoding at the destination to prove the following theorem.

Theorem 4: The rate tuple $\left(R_{1}, R_{2}, \ldots, R_{M}\right)$ is achievable for an $M$-source MARC if, for all non-empty sets $\mathcal{A}, \mathcal{G}, \mathcal{A} \subseteq$ $\mathcal{G} \subseteq \mathcal{S}$, we have

$$
\begin{aligned}
R_{\mathcal{G}, 2} & \leq I\left(V_{\mathcal{G}} ; Y_{M+2} \mid V_{\mathcal{G}^{c}}, Q_{\mathcal{S}}, X_{M+2}\right) \\
R_{\mathcal{G}, 1} & \leq I\left(X_{\mathcal{G}} ; Y_{M+1} \mid X_{\mathcal{G}^{c}}, V_{\mathcal{S}}, Q_{\mathcal{S}}, X_{M+2}\right) \\
R_{\mathcal{G}, 1}+R_{\mathcal{A}, 2} & \leq I\left(X_{\mathcal{G}}, X_{M+2} ; Y_{M+1} \mid X_{\mathcal{G}^{c}}, V_{\mathcal{A}^{c}}, Q_{\mathcal{A}^{c}}\right)
\end{aligned}
$$

where the joint probability distribution factors as

$$
\left[\prod_{m=1}^{M} p\left(q_{m}, v_{m}, x_{m}\right)\right] \cdot p\left(x_{M+2} \mid q_{\mathcal{S}}\right) \cdot p\left(y_{M+1}, y_{M+2} \mid x_{\mathcal{T}}\right) \text {. }
$$

Remark 5: The rate bounds for the HD-MARC are obtained by conditioning the bounds above on the relay's $L$ and $T$ states with probability $\alpha$ and $(1-\alpha)$ respectively.

For the Gaussian HD-MARC, consider proper complex Gaussian inputs. The resource allocation problem is then to optimize over the

- time fraction $\alpha$,

- power tuples $\left(P_{1}^{(H)}, P_{2}^{(H)}, \ldots, P_{M}^{(H)}, P_{M+2}^{(H)}\right)$,

- fractions $\gamma_{m, t}$ of power $P_{m}^{(H)}$ transmitted by the source $m$ in time slot $t$,

- fractions $\beta_{m, t}$ of power $\gamma_{m, t} P_{m}^{(H)}$ allocated by the source $m$ to transmitting $w_{m 1}$, and

- fractions $\alpha_{M+2, m}$ that the relay allocates to forwarding message $w_{m 0}$.

\section{COOPERATION vs. Hierarchy}

We consider networks with $M=2$ and with the planar geometry shown in Fig. 1. The sources are positioned symmetrically on the $y$-axis, and the destination is a unit distance

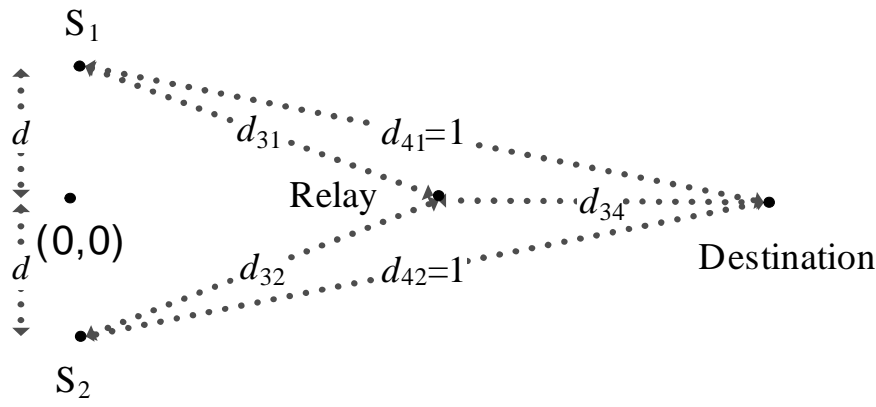

Fig. 1. Two-source geometry for HD-MAC-GF and HD-MARC

from both sources. For the HD-MARC, the relay is placed along the $\mathrm{x}$-axis between the source and the destination. We choose the path loss exponent $\gamma$ as 4 and plot the rates in bits per channel use. The individual and total power constraints are $P_{1}=P_{2}=4, P_{4}=6$, and $P_{t o t}=8$. We present and analyze the results separately for the three fading models mentioned in Section II-E.

\section{A. No Fading}

In Fig. 2, we plot the maximum sum rate $R_{1}+R_{2}$ achieved by the PDF strategy against the symmetric distance $d$ of the two sources from the origin for the HD-MAC-GF and HDMARC. We also plot the non-cooperative multi-access sum capacity (labeled as MAC) and the cooperative sum capacity achieved when all messages are known a priori at both sources (labeled as full cooperation). The HD-MAC-GF sum rate is maximized by $P_{m}^{(C)}=P_{m}=4, m=1,2$. For the HDMARC, the sum rate, maximized over all parameters and relay position, is plotted for several combinations of $P_{1}^{(H)}=$ $P_{2}^{(H)} \leq P_{1}$ and $P_{4}^{(H)} \leq P_{4}$ subject to $2 P_{1}^{(H)}+P_{4}^{(H)}=P_{t o t}$ where each curve is characterized by a different $P_{\text {ratio }} \triangleq$ $P_{4}^{(H)} / P_{\text {tot }}$.

Fig. 2 shows the effect of cooperation when the sources are closer to each other than to the destination. The sum rate decreases as the inter-source distance $2 d$ increases until it coincides with the MAC sum capacity. The maximum HDMARC sum rate is plotted for several choices of $P_{\text {ratio }}$. Fig. 2 also shows the rate gains that result from using a relay with non-zero, albeit small, power $P_{4}^{(H)}$ relative to HD-MAC-GF sum rate and MAC sum capacity. The gain is a direct result of the path-loss dependent channel model and optimal positioning of the relay. The curves for various relay powers also reveal the effect of sharing $P_{t o t}$ between source message transmissions and relay cooperation.

We remark that, because the total power is held constant, the two networks can also be compared by scaling the rates in Fig. 2 by $P_{t o t}$ to obtain the network utility in bits/Joule [12] that results from cooperation. 


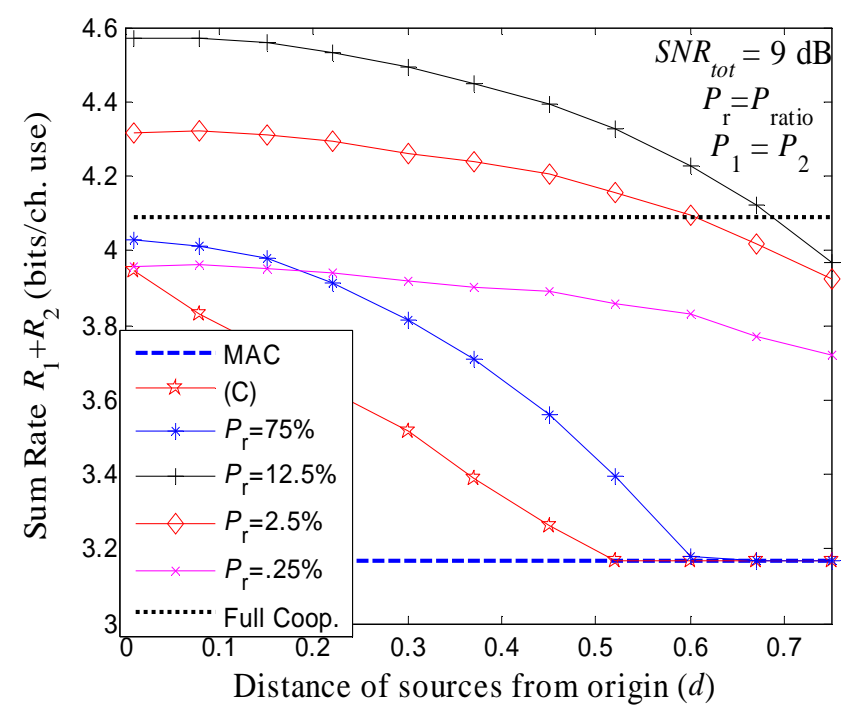

Fig. 2. Plot of $R_{1}+R_{2}$ for HD-MAC-GF and HD-MARC

\section{B. Ergodic phase fading}

For an ergodic fading channel with phase information unknown at the transmitter, the rates achieved for the DF and PDF strategies are maximized when the cooperating nodes transmit independently [5], [13]. Thus, since the source nodes cannot cooperate, the maximum sum rates for the HD-MACGF simplify to the MAC sum capacity. For the HD-MARC, rate gains relative to the MAC may be possible for certain choices of $\left(P_{1}^{(H)}, P_{2}^{(H)}, P_{4}^{(H)}\right)$.

The optimal HD-MARC sum rate curves for the no-fading case in Fig. 2 are obtained when $\beta_{m, T}=1$, i.e., when the relay and the sources transmit independently. Thus, these curves are the same as the curves for the ergodic phase-fading channel for this example. The maximum achievable HD-MAC-GF sum rate is given by the MAC sum rate in Fig. 2. This model further reveals the advantage of reducing source power while sharing the total power with a relay that is closer to the destination than the sources. Similar results can also be expected for ergodic Rayleigh fading channels.

\section{Quasi-static Rayleigh Fading}

We next consider the case where the channel parameters are chosen randomly but held fixed for all time. The best achievable rates are therefore random variables that are functions of the random channel parameters. We consider the above geometry with $2 d=0.5$, and set $R_{1}=R_{2}=R=.5$. We determine the probability $P_{\text {out }}$ that the two sources experience an outage. In Fig. 3, we plot $P_{\text {out }}$ for various models and resource allocations. We observe that half-duplexing the nodes to allow either source-source or source-relay cooperation can increase the spatial diversity at the destination, thereby decreasing $P_{\text {out }}$ for the two networks relative to the MAC. The advantage of source cooperation is clearly highlighted by the near-optimal performance of the HD-MAC-GF as compared to full cooperation.

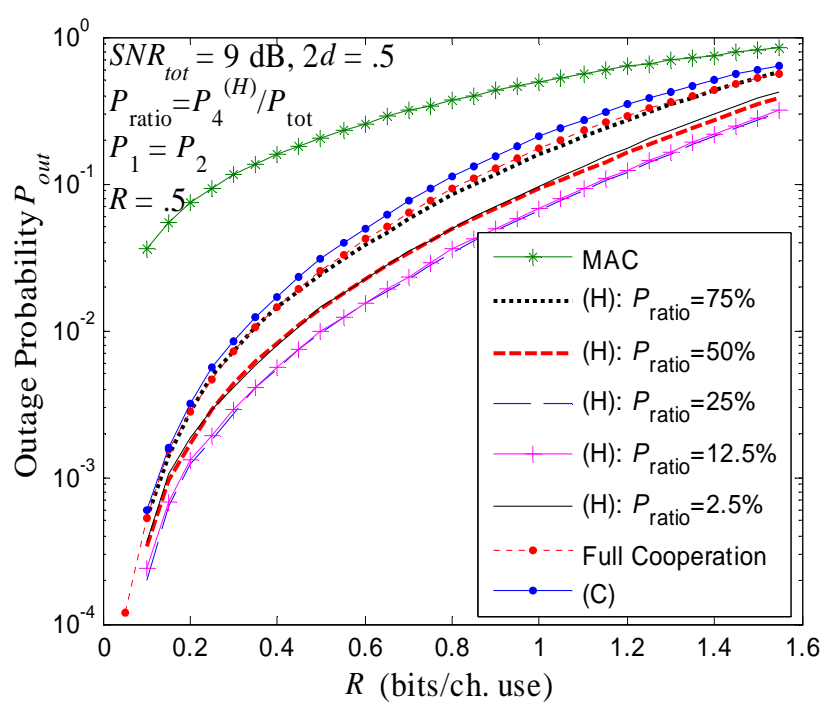

Fig. 3. Comparison of the outage probability for both networks at the symmetric rate point

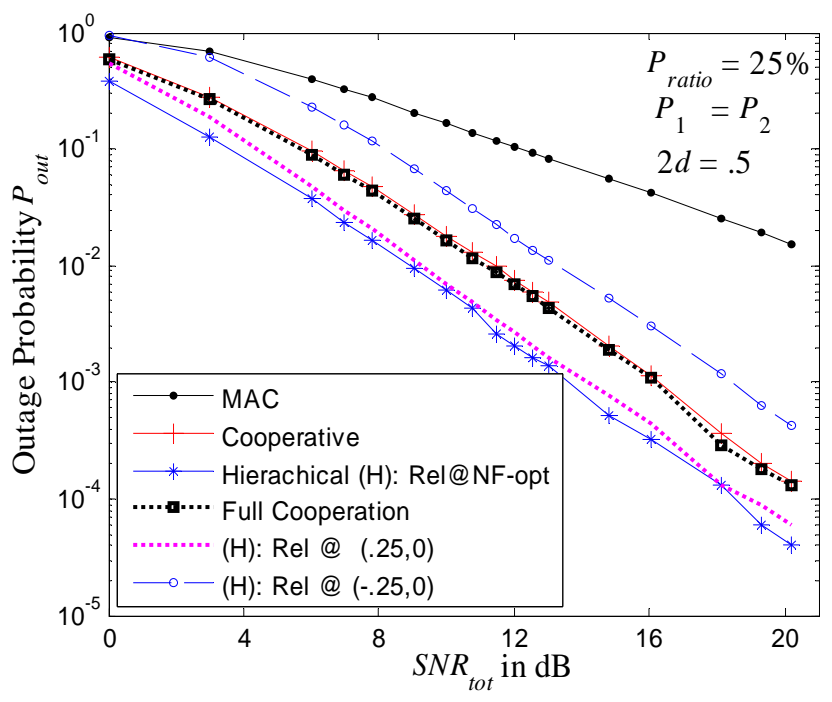

Fig. 4. Outage probability $P_{\text {out }}$ vs. total SNR $S N R_{\text {tot }}$

Fig. 3 also reveals a performance advantage of the hierarchical over the cooperative network. Quantifying this further, we define $S N R_{t o t}$ as the ratio of $P_{t o t}$ to the noise power at the destination. In Fig. 4, we plot $P_{\text {out }}$ against $S N R_{\text {tot }}$ for $R=$ $.5,2 d=.5$, and power constraints $P_{1}=P_{2}=P_{4}=P_{t o t} / 2$. For the two models, $P_{\text {out }}$ is plotted for $P_{m}^{(C)}=P_{m}, m=1,2$, and $P_{1}^{(H)}=P_{2}^{(H)}, P_{4}^{(H)}=P_{4} / 2$ subject to (4). We observe an increase in diversity from 1 to 2 for both models relative to the MAC. However, the SNR required for the HD-MARC is $2.5-3 \mathrm{~dB}$ lower than for the HD-MAC-GF (recall that we have positioned the relay to maximize the non-fading rate). Such gains in SNR are, in general, dependent on the relative position of the sources and relay, as evidenced by the outage curves in Fig. 4 for relay positions at $(.25,0)$ and $(-.25,0)$. 
We remark that the above analysis can be applied to other geometries, where the maximum diversity remains at 2 for $M=2$. For $M>2$, however, cooperative networks can achieve diversity gains of $M$, while hierarchical networks can achieve diversity gains of at most 2 (recall that, by assumption, the MARC source nodes can cooperate only with the relay). Comparable diversity gains in the hierarchical network require either multiple antennas at the relay or multiple relays. Finally, the above networks can also be compared when using the $\mathrm{AF}$ and CF strategies, a topic for future work.

\section{ACKNOWLEDGEMEnT}

We thank the anonymous referees for their valuable suggestions. L. Sankaranarayanan and N. B. Mandayam are supported in part by the NSF under grants CCR-0429724 and NeTS-0434854. G. Kramer is supported in part by the NSF under grant CCR-0325673.

\section{REFERENCES}

[1] O. Ileri, S.-C. Mau, and N. B. Mandayam, "Pricing for enabling forwarding in self-configuring ad hoc networks," IEEE JSAC, vol. 23, no. 1, pp. 151-162, Jan. 2005.

[2] A. Furuskar and K. Johansson, "An infrastructure cost evaluation of single- and multi-access networks with heterogeneous user behavior," in IEEE Vehicular Tech. Conf., Stockholm, Sweeden, May 2005.

[3] F. M. J. Willems, "Informationtheoretical Results for the Discrete Memoryless Multiple Access Channel," Ph.D. dissertation, Doctor in de Wetenschappen Proefschrift, Katholieke Universiteit Leuven, Leuven, Belgium, Oct. 1982.

[4] A. Sendonaris, E. Erkip, and B. Aazhang, "User cooperation diversity part I: System description," IEEE Trans. on Commun., vol. 51, no. 11, pp. 1927-1938, Nov. 2003.

[5] G. Kramer, M. Gastpar, and P. Gupta, "Cooperative strategies and capacity theorems for relay networks," Feb. 2004, accepted for publication.

[6] L. Sankaranarayanan, G. Kramer, and N. B. Mandayam, "Capacity theorems for the multiple-access relay channel," in 42nd Annual Allerton Conf. on Commun., Control, and Computing, Monticello, IL, Sept. 2004.

[7] — - "Hierarchical sensor networks: Capacity theorems and cooperative strategies using the multiple-access relay channel model," in First IEEE Conference on Sensor and Ad Hoc Communications and Networks, Santa Clara, CA, Oct. 2004.

[8] G. Kramer, "Models and theory for relay channels with receive constraints," in 42nd Annual Allerton Conf. on Commun., Control, and Computing, Monticello, IL, Sept. 2004.

[9] A. Host-Madsen and J. Zhang, "Capacity bounds and power allocation for the wireless relay channel," IEEE Trans. Inform. Th., vol. 51, no. 6, pp. 2020-2040, June 2005.

[10] J. N. Laneman, D. N. C. Tse, and G. Wornell, "Cooperative diversity in wireless networks: efficient protocols and outage behavior," IEEE Trans. Inform. Theory, vol. 50, no. 12, pp. 3062-3080, Dec. 2004.

[11] T. Cover and A. El Gamal, "Capacity theorems for the relay channel," IEEE Trans. Inform. Theory, vol. 25, no. 5, pp. 572-584, Sept. 1979.

[12] N. Feng, S.-C. Mau, and N. B. Mandayam, "Joint network-centric and user-centric radio resource management in a multicell system," Jan. 2003 , preprint.

[13] J. N. Laneman and G. Kramer, "Window decoding for the multiaccess channel with generalized feedback," in Proc. 2004 IEEE Int. Symp. Inform. Theory, Chicago, IL, June 27 - July 2, 2004. 